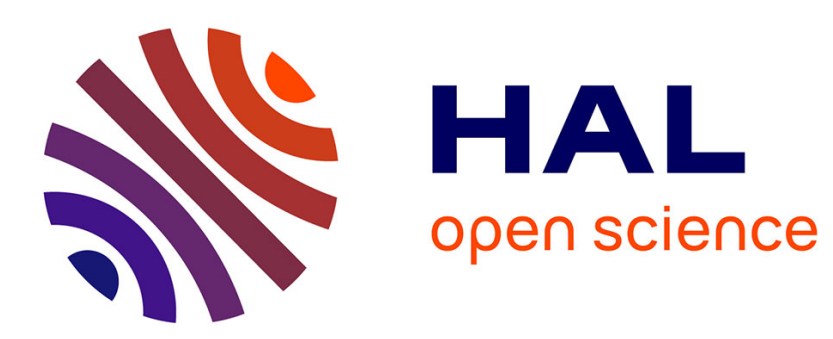

\title{
Observation psychiatrique et récit psychanalytique : l'écriture du cas en question
}

\author{
Thomas Lepoutre
}

\section{To cite this version:}

Thomas Lepoutre. Observation psychiatrique et récit psychanalytique : l'écriture du cas en question. Psychologie Clinique, 2017, 44, pp.72 - 85. 10.1051/psyc/20174472 . halshs-01728689

\section{HAL Id: halshs-01728689 \\ https://shs.hal.science/halshs-01728689}

Submitted on 11 Mar 2018

HAL is a multi-disciplinary open access archive for the deposit and dissemination of scientific research documents, whether they are published or not. The documents may come from teaching and research institutions in France or abroad, or from public or private research centers.
L'archive ouverte pluridisciplinaire HAL, est destinée au dépôt et à la diffusion de documents scientifiques de niveau recherche, publiés ou non, émanant des établissements d'enseignement et de recherche français ou étrangers, des laboratoires publics ou privés. 


\section{We Observation psychiatrique et récit psychanalytique : l'écriture du cas en question}

Thomas Lepoutre ${ }^{[1]}$

Résumé

On entend ici réinterroger les spécificités de l'écriture du cas en psychiatrie et en psychanalyse en repartant du grand constat, fait par Freud lui-même, que les " récits de cas " psychanalytiques tenaient plus du “ roman " que du “ certificat " psychiatrique. Il s'agit de penser cette originalité d'écriture en propre, en ce qu'elle fait cas du sujet, réalisant du même coup le déplacement du centre de gravité en cause dans “ l'observation psychiatrique ». Cela permet de dégager a contrario la logique du reproche freudien adressé au regard psychiatrique, régulièrement accusé par Freud d'être frappé d'une sorte d'interdit de voir, dès lors qu'il exclut le conditionnement inconscient du symptôme. Il y aurait là une sorte d'aveuglement médical devant le tableau clinique - la cécité contrastant d'autant avec le voyeurisme animant par définition une clinique du signe. Cela permet finalement d'envisager la posture freudienne devant le tableau clinique, telle que Freud l'a théorisée au détour de son analyse du “Moïse de Michel-Ange » en convoquant les travaux d'attribution scientifique du célèbre Giovanni Morelli. On souligne alors la portée de l'analogie existant entre cette méthode analytique inédite, et l'attitude proprement psychanalytique devant le tableau clinique.

Mots clés

Cas ; Kraepelin ; Morelli ; psychiatrie ; sémiologie ; tableau clinique.

\section{Summary}

In this context, we re-examine the specificities of the writing of the case in psychiatry and psychoanalysis, starting from the great observation made by Freud himself that the psychoanalytical " narratives of cases » were more a matter of the " novel » than of the psychiatric " certificate ». It is a matter of thinking about this originality of writing in its own right, in that it makes the case of the subject, thereby realizing the displacement of the center of gravity involved in " psychiatric observation ". This makes it possible to discern a contrario the logic of the Freudian reproach addressed to the psychiatric gaze, regularly accused by Freud of being struck with a sort of forbidden to see, since it excludes the unconscious conditioning of the symptom. There would be a sort of medical blindness in front of the clinical picture - blindness contrasting with voyeurism by definition, a clinic of the sign. This finally allows us to consider the Freudian posture in front of the clinical picture, as Freud has theorized it in the bend of his analysis of the "Moses of

[1] Maître de Conférences, Université Aix-Marseille, LPCLS (EA3278), psychologue clinicien, Paris. thomaslepoutre@free.fr 
Michelangelo " by summoning the works of scientific attribution of the famous Giovanni Morelli. The significance of the analogy between this unpublished analytical method and the properly psychoanalytic attitude towards the clinical picture is emphasized.

Key words

Case ; clinical frame ; Kraepelin ; Morelli ; psychiatry ; semiology.

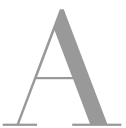

u moment de réinterroger les spécificités de l'écriture du cas en psychiatrie et en psychanalyse, et pour situer précisément l'originalité du récit psychanalytique en regard de l'observation psychiatrique, on peut repartir d'un événement significatif pour le fondateur de la psychanalyse : il s'agit de sa première expérience en psychiatrie, dont il convient de dégager la portée au plan de l'écriture (freudienne) du symptôme.

\section{W "L'empreinte de sérieux de la scientificité... "}

Il se trouve que le 26 avril 1883, pour parachever son internat, Sigmund Freud s'installe dans le service psychiatrique de l'Hôpital général de Vienne, dirigé alors par Theodor Meynert, où il réalise ainsi son premier stage en psychiatrie, d'à peine plus de cing mois (il en repartira le $1^{\mathrm{er}}$ octobre 1883$)^{[2]}$.

D'une façon étonnante, on trouve un relatif silence biographique sur cet épisode. Et pourtant, si cette expérience de Freud en psychiatrie est à l'origine relativement étroite, elle n'en est justement que plus déterminante sur l'imaginaire du futur fondateur de la psychanalyse. C'est en effet là que Freud s'est forgé une idée du quotidien du psychiatre, là qu'il s'est construit sa représentation de la profession et de son travail clinique; c'est là, aussi, que Freud a rencontré et fréquenté quotidiennement la psychose - ce pourquoi cette expérience limitée garde l'importance cruciale des premières fois.

Il y va alors, pour Freud, d'un apprentissage d'une certaine écriture de la clinique. Nous restent de fait de cette expérience originaire une centaine de dossiers de patients rédigés de la main de Freud lui-même, rendus publics grâce aux travaux d'Albrecht Hirschmüller (1991).

Nous voilà donc face à une source de premier ordre, pour juger d'un peu près cette première découverte freudienne de la clinique psychiatrique. Le dépouillement et l'analyse de leur contenu autorisent une série d'observations que l'on peut schématiser comme suit :

- C'est d'abord la contrainte déjà codifiée de l'écriture psychiatrique qui se donne à lire : état civil, date d'entrée, âge, religion, situation matrimoniale, durée d'hospitalisation, diagnostic, données pour les statistiques (hérédité, état somatique, tableau symptomatologique, mode évolutif et état terminal, nombre de rechutes, traitements).

[2] Voir à ce sujet Freud et Bernays, 2011. Un dossier de patient annoté de la main de Freud en date du 24 avril 1883 suggère qu'il a pu commencer plus tôt. 
Suivent les observations libres proprement dites, habituellement de deux ou trois lignes par jour, maximum.

- Or ceci précisé, il nous faut aussitôt souligner que les dossiers médicaux ne manifestent, à quelques exceptions près, qu'une faible précision dans le relevé sémiologique, ne comportent presque aucune discussion diagnostique et, dans l'ensemble, incluent la plupart du temps des anamnèses extrêmement sommaires. Tout se passe comme si la plupart des dossiers avaient été remplis par Freud "à la va vite », d'où il ressort l'impression d'une franche négligence dans le maniement des concepts psychopathologiques. Cela suggère une tension travaillant le cour de la psychiatrie universitaire, à laquelle Freud n'échappe pas à l'époque, entre l'imprécision des diagnostics et l'approximation de la réflexion psychopathologique dans la clinique d'une part, et l'exactitude et la méticulosité du travail de laboratoire, penché audessus du microscope, d'autre part.

- Finalement, c'est la posture clinique de Freud, du moins telle qu'elle qu'on peut en juger à travers ses notes, qui mérite d'être relevée: lorsqu'il fait fonction de psychiatre dans ces années précoces, Freud ne semble pas particulièrement s'investir dans la rencontre thérapeutique et ferait plutôt preuve d'une distance vis-à-vis des patients psychotiques, ce qui ne le distingue en rien de ses collègues d'alors.

Quelles conclusions peut-on tirer d'un tel coup d'œil jeté sur la première écriture psychiatrique de Freud? En vertu de la brièveté de ses descriptions cliniques, l'absence de diagnostics différentiels, son mutisme biographique pratiquement complet, et l'insistance sur les conduites à tenir, on peut y discerner une découverte de la clinique psychiatrique plutôt peu enthousiasmante, et inviter à conclure, avec Hirschmüller, à l'insatisfaction de Freud en position de psychiatre. Aussi a-t-on volontiers parlé d'une rencontre manquée de Freud avec la psychiatrie, qui expliquerait pourquoi il “ n’est pas devenu psychiatre "(Hirschmüller, 1991, p. 14), en majorant le manque d'intérêt de Freud et en alourdissant ainsi la déception d'origine. Mais l'enjeu n'est pas de décider si Freud aurait fait un bon psychiatre, ce qu'il n'a manifestement jamais envisagé. Il s'agit plutôt de constater que lorsque Freud s'essaye en psychiatre, il ne se démarque pas de ce qui se pratique alors: si ses enquêtes anamnestiques s'avèrent incomplètes, si ses descriptions cliniques sont grossièrement brossées, si ses diagnostics sont peu argumentés, c'est que, pour le psychiatre de la fin du XIX ${ }^{e}$ siècle, l'écriture de la scientificité se joue ailleurs - dans le laboratoire d'anatomo-pathologie.

En revanche, on peut alors pleinement confirmer que pour s'être plié aux contraintes d'un dossier de soin pré-rempli, Freud sait parfaitement ce qu'est une observation psychiatrique en bonne et due forme.

Or il convient de s'aviser qu'au moment où il forge sa clinique, la clinique psychanalytique, et qu'il commence à transcrire les grands récits de cas que l'on connaît, c'est justement ce souvenir qui rebondit vers le psychanalyste qu'il est devenu, en lui imposant de constater l'originalité de l'écriture clinique particulière qu'il inaugure. 
C'est dans les Études sur l'hystérie qu'on en trouve le témoignage, célébrissime, le plus probant : “Cela ne cesse de me faire à moi-même une impression singulière de voir que les histoires de malades que j'écris se lisent comme des romans et sont pour ainsi dire privées de l'empreinte de sérieux de la scientificité. » (Freud, 1895, p. 182). On a souvent attiré l'attention sur l'aveu par Freud du malaise inhérent à une telle mutation de l'écriture de cas, imposée par la psychanalyse (Assoun, 1987) : c'est que découvrant l'effet de parole du symptôme lié à sa surdétermination historique, le fondateur de la psychanalyse se sent immanquablement transporté, par cette parole même, en dehors du sérieux sans cesse revendiqué par la scientificité, vers le narratif de l'histoire de malade. En imposant la nécessité d'une narration tenant alors plus du roman que du certificat, c'est donc justement cette démarcation d'avec ses observations psychiatriques de jadis que le récit de cas freudien rappelle immanquablement. Or entre la scientificité du dossier codifié et le romanesque des analyses freudiennes, il n'y a pas qu'un changement de style.

D’abord en vertu du “ noyau narratif »(Kohn, 1998) en psychanalyse, inévitablement lié à une prolifération des détails biographiques absolument vertigineuse en regard de l'univoque codage diagnostique ayant cours en psychiatrie. Freud l'a lui-même reconnu : il y va, en psychanalyse, d'une “ intrigue du symptôme » nécessairement évocatrice du “ roman à clés » (Freud, 1905, p. 189).

Le problème vient de ce que les innombrables détails du récit de cas freudien, par leur apparent manque de sérieux et leur caractère éminemment contingent, menacent toujours la scientificité de l'histoire qu'ils envahissent - et pour ainsi dire sa crédibilité psychiatrique. Naomi Schor soulignait très judicieusement, à ce sujet, comme faisait problème pour la psychanalyse " le statut du détail écrit (dans le récit de cas) par opposition au détail parlé (dans la séance) »-situation paradoxale du détail qui fait que, surgissant comme moment de vérité traversant le sujet dans la séance, il apparaît pourtant s'inscrire comme en faux en dehors de la séance, et notamment dans le texte scientifique, à destination du psychiatre. L'incrédulité du lecteur-psychiatre naîtrait de cette “ loi du détail » organisant et encombrant l'écriture clinique, loi paradoxale telle que “ disséminateur de fiction d'un côté, le détail est [pourtant] révélateur de vérité de l'autre »(Schor, 1980, p. 5).

Or il faut en réalité prendre acte de cette valeur pour ainsi dire romanesque du récit de cas détaillé, qui vient en effet à caractériser la clinique freudienne, tout en faisant cas de la revendication simultanée par Freud du titre de science pour la psychanalyse : c'est que la clinique impose elle-même cette narration inédite. Certes, cela suppose toujours “ une présentation approfondie des processus animiques, comme on a l'habitude d'en trouver chez le poète ". Mais en réalité, que le cas s'écrive comme un récit, cela ne fait que mimer la logique du symptôme même : c'est que celui-ci convoque spontanément, dans le dire, une histoire détaillée qui se prête à l'écriture de la clinique. C'est ce qui donne au récit de cas freudien, comme à la clinique analytique, son sceau particulier : “ De telles histoires de malades doivent être jugées 
comme des histoires psychiatriques, mais elles ont sur ces dernières un avantage, à savoir la relation intime entre l'histoire de souffrance et les symptômes de maladie, celle-là même que nous cherchons en vain dans les biographies d'autres psychoses. » (Freud, 1895, p. 182).

L'histoire de souffrance se trouve brusquement promue dans le regard analytique et impose la narration, à l'intérieur de laquelle les symptômes de maladie surgissent comme des événements - seuls phénomènes supposés jusque-là dignes de la scientificité psychiatrique comme telle. Mais justement, ce qu'opère Freud, c'est précisément le déplacement du centre de gravité de la scientificité en cause dans l'observation psychiatrique, en faisant cas du sujet - en ceci que l'histoire de souffrance fournit en réalité le ressort d'intelligibilité du symptôme de maladie.

C'est en effet précisément l'un des vides caractéristiques des dossiers psychiatriques, que Freud connait bien pour les avoir lui-même rédigés à une époque, qui se trouve ainsi visé et pour ainsi dire corrigé par cette originalité d'écriture. Entendons que la prolixité de l'analyste sur les détails de l'histoire du cas peut bien être jugée superflue par la rationalité psychiatrique et générer un effet fiction pour le lecteur psychiatre, c'est pourtant bien la détermination historique du symptôme jusqu'en ses “ détails les plus fins "(Freud, 1895, p. 112) qui l'impose, au-delà de la tactique narrative, pour le lire comme un effet de réel. Par un étonnant renversement, c'est alors finalement le récit psychanalytique qui adresse son reproche à la scientificité de l'observation psychiatrique, en accédant à son ambition propre. Voici le blanc, le trou, que l'écriture du cas freudien entendra corriger : rapporter en sa complexité l'histoire de souffrance qui fournit le ressort du symptôme pour articuler clairement leur relation intime - celle que l'écrit psychiatrique, en sa scientificité irréprochable, s'obstine à méconnaître, sinon même s'emploie à rendre illisible...

C'est ce en quoi l'empreinte de sérieux qui habille bel et bien la scientificité des observations psychiatriques pourrait bien s'avérer, selon Freud, relever en réalité d'un " semblant d'exactitude » [Scheinexaktheit]. À quoi doit répondre, en fait, le récit exhaustif de ce qui n'est justement habituellement pas dit, de ce qui est tu, dans l'anamnèse psychiatrique courante, laquelle passe complaisamment sous silence, sous couvert d'objectivité, ce chapitre censuré de l'histoire du sujet qui fait l'inconscient.

A contrario, la nécessité des détails revient-elle pour autant, dans l'écriture d'un cas, à tout écrire, comme il convient, dans la séance, de tout dire? Pas nécessairement. Ainsi Freud révoque-t-il dans l'écriture de ses cas, par exemple, le compte rendu sténographique permettant d'assister par procuration à l'intégralité des séances de l'analyse. “ Dans une histoire de malade analytique, des procès-verbaux précis apportent moins que ce que l'on pourrait attendre d'eux. Ils appartiennent, rigoureusement parlant, à cette exactitude apparente [Scheinexaktheit] dont la psychiatrie "moderne" nous offre plus d'un exemple frappant. "(Freud, 1912, p. 148). 
On vérifie là que Freud suspecte volontiers le compte rendu exhaustif et innocemment transcrit, comme expédient au service de la communicabilité scientifique, pour le récuser en son principe même, si tant est que son exactitude exhaustive tait ce dont il est réellement question. En réalité, écrire, puisqu'on ne peut tout écrire, c'est toujours choisir. Comment choisir, donc, ce qui doit être écrit?

\section{\$E Cécité psychiatrique et voyeurisme sémiologique}

Cela suppose d'abord, pour Freud, de savoir regarder le symptôme en face. Entendons que pour écrire le symptôme, pour qu'un symptôme soit scriptible, il faut que celui-ci soit lisible - et même visible. C'est le reproche majeur adressé par Freud à la clinique psychiatrique - celui qui doit nous permettre de dégager, a contrario, l'originalité que Freud revendique pour la posture analytique, à l'égard de la position commune du psychiatre.

L'occasion en est une remarque privatim à Jung, rapportant une visite de l'asile de Görlitz, durant laquelle Freud rencontre un schizophrène présentant, sur le mode d'une stéréotypie spécialement évocatrice, un symptôme spécialement éloquent - c'est Freud qui rapporte : “ À présent un accès se présente ainsi : il (c'est le patient) se tient debout devant une porte, crie, hurle, rage et crache. Si on contemple la scène on voit au premier coup d'oeil - mais un véritable psychiatre ne doit pas voir ce qui n'est pas dans Kraepelin - qu'il frotte avec deux doigts de la main droite un sillon du panneau de la porte, en un mouvement montant et descendant (c'est ce que j’ai vu !), c'est-à-dire qu'il imite un coït! „(Freud et Jung, 1975, p. 80-81).

“Un véritable psychiatre ne doit pas voir ce qui n'est pas dans Kraepelin » : si pour Freud la posture kraepelinienne emblématise la posture psychiatrique toute entière, telle est la formulation critique du point de désaccord le plus manifeste entre psychanalyse et psychiatrie - la formule freudienne concentrant ironiquement les divergences dans les façons de voir le pathologique.

Que la psychiatrie se constitue pour ne pas voir ce qui s'impose pourtant au premier coup d'œil, voilà le paradoxe stupéfiant donnant à penser que l'irréprochable scientificité de l'observation psychiatrique trouve son meilleur aboutissement dans ce déni professionnel du sens - sexuel, de surcroît.

C'est ce qui ouvre finalement sur cet essentiel enjeu ad hominem qui infiltre bien la spécialisation en psychiatrie - soit le fait qu'elle ait fort affaire avec la résistance personnelle du psychiatre, le conduisant chroniquement, selon Freud, à fermer les yeux sur l'effet de sens surdéterminant le symptôme.

Cela renvoie en outre à une forme d'aveuglement médical devant le tableau clinique, le psychiatre n'acceptant jamais d'y retrouver effectivement que le symptôme typique qui a été homologué par ailleurs, comme pour reconnaître ce qui est en fait connu d'avance, déjà donné, mais non ce qui est inédit dans le cas individuel. D'où cet interdit de voir qui frappe en quelque manière de cécité le regard psychiatrique 
- quitte à produire cette sorte de scotome au coeur du tableau clinique aboutissant à exclure le conditionnement du sens sur le symptôme individuel. Il y aurait là une sorte de tabou du regard psychiatrique - la cécité contrastant d'autant avec le voyeurisme animant par définition une clinique du signe.

À ce sujet, notons de façon incidente que ce reproche de Freud adressé au regard psychiatrique est en fait d'autant mieux motivé qu'une conscience historique minimale nous rappelle que Kraepelin est rigoureusement contemporain et partie prenante d'un vaste mouvement de transformation architecturale de l'asile, visant justement à y établir le " principe d'une observation permanente ${ }^{[3]}$ - c'est l'époque de la mise en place des “ salles d'observation " [Wachabteilung] (voir par exemple Kraepelin, 1895, 1901 et 1902), l'augmentation du personnel de surveillance et des équipes de nuit, la mise en place des transmissions infirmières, autant d'éléments permettant à l'œeil du psychiatre de s'immiscer dans les hors-champs et les hors-scènes cliniques, et garantissant la continuité d'un regard psychiatrique pouvant tout voir et intervenir rapidement.

Or contre cette institutionnalisation du regard psychiatrique en son genre panoptique, Freud préfère le “ coup d'oeil » [Kück] (Freud, 2007, p. 190) dont l'acuité clinique met le fait bien en évidence, et l'isole dans des conditions où le phénomène parle. Là où Kraepelin promeut l'extension du voyeurisme psychiatrique aux espaces interstitiels, pour contourner l'éventuelle réticence du malade à livrer sa vérité, Freud recentre l'écoute sur ce qui se dit et ce qui se donne à voir hic et nunc, pour affronter la résistance - quitte à assumer de s'en tenir, pour son jugement, au seul moment offert au transfert, et à ce que le patient lui montrera in praesentia, le temps de la rencontre clinique.

Double évidence qui anime la clinique freudienne, en raison de sa foi méthodologique envers “ la rigueur du déterminisme des processus animiques » (Freud, 1910, p. 26) : évidence que tout ce qui surgira en face de l'analyste aura nécessairement un rapport avec ce qu'on cherche ; et évidence parlante du fait ainsi mis juste sous les yeux : ce que le sujet cache de sa vérité n'a pas lieu dans le dos du psychiatre, mais précisément sous son regard, simplement d'une façon déformée.

C'est d'ailleurs en ce sens qu'on trouve chez Freud, contrairement à ce que l'on dit trop souvent, une préoccupation tout à fait marquée pour le détail de la sémiologie. " Je pourrais ici éveiller l'impression d'accorder trop de poids aux détails des symptômes et de me perdre dans une manie superflue d'interprétation des signes. Cependant j'ai appris que la détermination des symptômes [...] descend réellement jusqu'à leurs détails les plus fins et que l'on ne saurait guère leur imputer trop de sens. » (Freud, 1895, p. 112).

Restituer dans leurs droits les détails du symptôme : telle est la stratégie clinique inséparable de l'entreprise analytique, consistant à supposer l'inconscient là où

[3] Voir en particulier les pages consacrées à la « Réorganisation de la clinique de Heidelberg par Kraepelin » dans Engstrom, 2003, p. 135-144. 
n'apparaît tout d'abord que l'arbitraire ou l'énigmatique du symptôme. C'est ce qui explique que la clinique psychanalytique ne puisse pas se réduire à un pur et simple "musée " ${ }^{[4]}$ de faits psychiatriques, soit une succession de tableaux que le diagnostic viendrait grossièrement légender. Encore faut-il repérer la place qu’y tient le symptôme, l'envisager du point de vue des relations systémiques qui l'insèrent dans le détail d'une telle composition, et en ressaisir les règles d'agencement.

Aussi faut-il le noter: s'il est arrivé à Freud de décrire les névroses comme ces " grandes œuvres d'art de la nature psychique»(Freud et Jung, 1975, p. 317), ce n'était pas pour encadrer le symptôme - comme y tend une certaine tradition clinique visant à collectionner les beaux faits mis en exergue dans le patrimoine de l'expérience psychiatrique - mais bien plutôt pour souligner que le tableau clinique impose au clinicien le dur travail d'en percer les lois de composition, jusqu'en ses détails les plus fins - d'où l'élargissement du regard.

Mais alors, cela suppose de faire droit non seulement aux signes " cardinaux », pour reprendre le mot de Kraepelin, soit ceux qui obsèdent le voyeurisme sémiologique de la clinique psychiatrique, mais également à l'irremarqué dans le tableau clinique. Quitte à insister, à l'occasion, sur une vétille pour le regard psychiatrique : “C'est vrai, la psychanalyse ne peut se glorifier de ne jamais s'être occupée de vétilles. Bien au contraire, la matière de ses observations est communément constituée de ces événements imperceptibles qui sont mis à l'écart par les autres sciences comme étant par trop infimes, et sont pour ainsi dire le rebut du monde des phénomènes. „(Freud, 1916-17, p. 20).

C'est bien le fonds commun de la sémiologie psychanalytique : l'événement imperceptible, le rebut du monde des phénomènes, les petits faits qui sont habituellement intraitables par le regard médical : autant de données marginales ravalées au rang de déchet de l'observation psychiatrique, et justement dénoncées comme contingentes par un Kraepelin - s'agissant de donner à voir la forme épurée du processus morbide - et pourtant considérées comme révélatrices par Freud - s'agissant de donner à penser le sens du symptôme.

\section{$\checkmark$ Le « rebut de l'observation »: Freud devant le tableau clinique}

Cela permet finalement d'envisager la position propre du psychanalyste, si différente de celle du psychiatre, face à l'énigme du symptôme - symptôme qui constitue “ l'une des questions cruciales du malentendu entre psychiatrie et psychanalyse » Vanier, 2005). Avec le symptôme, on touche en effet au cour de la conjonction psychanalyse

[4] On se souvient comme Freud entendait ouvrir une "Introduction à la psychanalyse » : " Même en psychiatrie, la présentation clinique du malade vous apporte, d'après ses changements de physionomie, sa façon de parler et son comportement, quantité d'observations qui vous laissent de profondes impressions. Ainsi, le professeur de médecine joue avant tout le rôle d'un guide et commentateur, qui vous accompagne à travers un musée tandis que vous acquérez une relation immédiate aux objets et que vous croyez vous être convaincus par votre propre perception de l'existence des faits nouveaux. II en va malheureusement tout autrement en psychanalyse. » (Freud, 1916-17, p. 11). 
et psychiatrie : on y trouve en forme de noud cette question essentielle de la hiérarchisation des signes, solidaire de la marginalisation du sens.

Or sur ce point, on ne l'a pas assez mis en valeur mais Freud a bel et bien théorisé à sa façon la posture de l'analyste devant le tableau clinique, au détour de son analyse du “ Moïse de Michel-Ange ». C'est ainsi que, pour se situer par rapport à la clinique psychiatrique et pour ainsi dire picturale, il a pu convoquer les travaux d'attribution scientifique d'un certain Ivan Lermolieff, en réalité le célèbre Giovanni Morelli, médecin italien et amateur d'art éclairé, et souligner la profonde solidarité existant entre sa méthode analytique inédite (consistant à détourner le regard de l'impression d'ensemble [Totaleindruck] pour mettre en relief les détails de la peinture), et l'attitude proprement psychanalytique devant le tableau clinique.

Face au tableau, pour distinguer en toute sûreté les copies des originaux et construire à partir des œuvres libérées de leurs assignations antérieures une vaste révision dans l'attribution de nombreuses toiles à tel ou tel peintre, Morelli avait fait à son époque sensation en “ enjoignant de faire abstraction de l'impression d'ensemble et des grands traits d'une peinture et soulignant la significativité caractéristique de détails secondaires, de petits riens ». Or Freud remarque que ce “ procédé est fort apparenté à la technique de la psychanalyse médicale. Cette dernière, elle aussi, est habituée à partir de traits tenus en piètre estime ou non remarqués, à partir du rebut - du "refuse" - de l'observation, à deviner ce qui est secret et ce qui est caché. » (Freud, 1914, p. 143).

La comparaison est éloquente : la clinique freudienne se constitue toujours à partir d'un rebut, d'un refuse, qui tend à exclure du champ du regard le détail significatif (c'est-à-dire rien de moins que le fait lui-même), quitte à promouvoir contre le jugement psychiatrique un trait jusque-là tenu en piètre estime. Cela nous rappelle que l'observation clinique est elle-même travaillée par la résistance, qui se prend à l'effet esthétique du tableau d'ensemble pour ne pas voir - horribile visu! - l'indice éventuellement le plus déterminant. Mais voilà, Freud refuse que l'on ajoute ou que l'on supprime dans le tableau ce que nous voulons ou ne voulons pas y trouver - d'abord pour ceci que l'on ne sait jamais d'emblée ce qui fera savoir dans le cas.

C'est ce qui impose cette sorte de déconstruction analytique du tableau, allant contre la fascination aveuglante de l'impression d'ensemble, contre les grands traits des peintures psychologiques (celles ayant constitué au fil des temps la vaste galerie de portraits de la psychiatrie), pour y mettre en lumière le relief négligé. Ainsi Freud isole-t-il l'indice masqué au regard, inscrit à la marge, refoulé au second plan, mais pourtant seul véritablement décisif : point aveugle du tableau, qui n’en fait pas moins trait du cas d'être laissé dans l'ombre. Du reste, laissé dans l'ombre est mal dire, car ce détail n'est pas enfoui, il n'émerge pas, informe, du fond du tableau. Il est même, comme l'était “ le dessin des ongles des doigts, des lobes de l'oreille, de l'auréole » dans la "clinique " de Morelli, ce qu'il y a de plus strictement déterminé dans le tableau - c'est à ce titre qu'il fait support au sujet, dans le cas, comme ce qui faisait support à l'auteur, dans l'œuvre. 
En fait, de la même manière qu'un criminel se trahit par les empreintes digitales qu'il laisse inconsciemment, comme sa signature, sur la scène du crime ${ }^{[5]}$, il y a des détails qui trahissent la présence d'un artiste donné sur la scène de l'œuvre : et quand bien même un tel détail peut avoir quelque chose de trivialement rebutant pour qui se complait dans la contemplation du tableau d'ensemble, il reste que le signe le plus discret peut bien être subjectivement le plus décisif.

C'est pourquoi Morelli se défendait ironiquement (avec une ironie qui n'était certes pas faite pour déplaire à Freud) contre ses adversaires " qui se complaisent à me prendre pour un homme incapable de voir le sens spirituel d'une œuvre d'art, donnant pour cette raison une importance particulière accordée à des signes extérieurs, tels que les formes de la main, de l'oreille et même, horribile dictu, d'une chose aussi antipathique que les ongles » (Morelli, 1994, p. 102). En réalité, il n'y a dans le tableau rien d'esthétiquement neutre, de la même manière qu'il n'y a dans le symptôme rien de subjectivement superflu : dans la clinique morellienne, toutes ces données marginales sont spécialement révélatrices, parce qu'elles témoignent des moments de vigilance où l'artiste, lié par la tradition culturelle, se relâche pour laisser place à l'expression de traits purement personnels qui lui échappent, et qu'il introduit dans l'œuvre “ sans y porter attention ou même sans s'en apercevoir, et souvent là où ils n'ont rien à faire. » (Morelli, 1994, p. 163).

On voit que l'originalité de la méthode tient justement dans ce postulat : c'est dans ces " minuties matérielles », ces éléments soustraits au contrôle de la conscience artistique et échappant à l'intention du projet esthétique, que l'on retrouve la dispersion des marques les plus caractéristiques de la subjectivité - moyennant quoi la personnalité de l'artiste se laisse identifier “ là où l'effort personnel est le moins intense »(Wind, 1989, p. 65). Le détail, par les formes de prédilections qui s'y livrent, renferme la signature subjective de l'artiste et trahit sa présence dans l'œeuvre d'une façon beaucoup plus caractéristique que tous les grands traits typiques qui sont certes communément considérés comme importants d'un point de vue artistique (composition, proportion, couleur, expression, geste, etc.), mais qui sont justement à la portée de n'importe quel imitateur.

Ainsi donc, si dans sa démarche d'attributionnisme Morelli concentrait son attention sur ce que « le copiste néglige d'imiter et que pourtant tout artiste exécute d'une marnière qui le caractérise ", c'est que c'est dans ce rebut inesthétique, ce détail de style que s'inscrit l'auteur, marquant au coin de son ouvre comme une marque de fabrique qui permet de l'authentifier. Ex ungue leonem - de même que c'est à sa griffe qu'on reconnaît le lion, c'est dans le détail différentiateur que se signe la marque précise d'un maître.

[5] Voir à ce sujet Ginzburg, 1980. 
D'où le paradigme indiciel (Ginzburg, 1980) de cette méthode empirique : s'appuyer non sur les traits typiques, mais sur les indices inesthétiques par lesquels chaque peintre appose ses conditions individuelles sur le tableau d'ensemble.

Entendons que pour identifier la main du maître puis la distinguer de celle d'un copiste, si l'on peut s'appuyer de façon privilégiée sur les idiosyncrasies, les traits secondaires insignifiants, dont les caractéristiques échappent à l'attention d'un faussaire ou d'un imitateur... c'est justement parce qu'elles échappent à celle de l'auteur lui-même, rattrapé et devancé par ses propres idiosyncrasies, et ses propres automatismes - fournissant autant de signatures cachées dans un motif latéral du tableau. On est alors en mesure d'enregistrer la profonde affinité avec la méthode proprement psychanalytique : c'est précisément cette rentabilisation du détail que repère l'analyste, travaillant comme l'historien de l'art, dans le tableau clinique : partout Freud invite en effet à " prendre en considération un détail qui n'a l'air de rien " (Freud, 1916-17, p. 260) dans le regard psychiatrique - pour autant que c'est dans ce détail idiosyncrasique du tableau que se retrouvera la marque inaliénable du sujet. D'où la déconstruction de l'objet qu'il a en face de lui, pour savoir où s'y livre le sujet. On rejoint là, du reste, la rationalité proprement freudienne, qui suppose toujours l'analyse et jamais la synthèse - en quoi il y a en psychanalyse, non pas simplement construction du cas (Villa et Fédida, 1999), mais d'abord et avant tout déconstruction du cas.

Comment s'opère une telle déconstruction du tableau, dans l'expérience clinique? Freud nous l'indique : si le symptôme porte à l'expression (en son genre artistique) le rapport divisé du sujet à sa propre vérité, une telle décomposition suppose de le “ défaire par le récit» (Freud, 1895, p. 54).

\section{W Portrait psychiatrique et récit psychanalytique}

On comprend que, là où l'observation psychiatrique apprend à négliger méthodologiquement les signes individuels et contingents pour laisser apparaitre la forme essentielle, et faire en quelque sorte le portrait épuré du processus morbide, Freud confesse (ne) s'attacher (qu')aux détails les plus insignifiants de tableau, pour y révéler le trait du cas, soit ce qu'il y a de plus individuel dans le sens du symptôme.

Le recours à la méthode morellienne sert alors à formuler la théorie de ce déchiffrage singularisant. Qu'on s'en avise en se tournant vers les Leçons d'introduction à la psychanalyse: on retrouve au cœur de la clinique freudienne ce même partage, cette même tension dans l'œuvre de la névrose, entre ce qui est absolument propre à chacun, donnant une symptomatologie marquée par des traits individuels - symptomatologie qui se relie en quelque sorte spontanément à une détermination historique - et des symptômes typiques, beaucoup plus communs, et pour ainsi dire anonymisés, sur lesquels certes “ nous nous orientons pour établir le diagnostic », mais pourtant relativement dévalués par l'analyste parce que beaucoup plus rebelles à 
l'interprétation, pour autant que “ les différences individuelles disparaissant entre eux, ou du moins se réduisant à tel point, il est difficile de les mettre en relation avec l'expérience individuelle de vie des malades et de les rapporter à telle ou telle situation vécue. » (Freud, 1916-17, p. 279).

On le voit, là où toute la rationalité psychiatrique commande une certaine posture anonymisante devant le symptôme, aboutissant, par un processus de sélection, à privilégier les symptômes cardinaux et dévaluer les symptômes individuels, Freud est lui hautement sensible à cette tension par laquelle l'idiosyncrasique du symptôme conteste toujours le typique de la symptomatologie (voir Lepoutre et Villa, 2016). Dans l'ordre de ses priorités, Freud aurait en réalité même tendance à inverser le sens de cette préférence psychiatrique, en privilégiant la part des symptômes appelés à chiffrer un sens absolument particulier, sens qui n’est jamais commun, " pas même propre à chaque structure " pour reprendre le mot de Lacan, et en se détournant un peu de celle des symptômes typiques, réguliers, à ce titre évocateurs pour le diagnostic mais absolument muets quant à la diversité des vérités individuelles. Sauf à considérer qu'il n'y a du reste, à bien y regarder, pas de symptôme typique qui ne se singularise en quelque endroit - ce pourquoi la clinique analytique, en regard de la clinique psychiatrique, se spécifie d'être un pur éloge de la singularité, visant alors à dénicher " suffisamment de traits individuels pour rendre possible l'interprétation pour ainsi dire historique »(Freud, 1916-17, p. 279).

De ceci ressortent néanmoins nettement ces deux directions opposées de l'écriture du cas où s'engagent psychanalyse et psychiatrie, de par leur profession de foi méthodologique. À un pôle plutôt nomothétique, la psychiatrie ambitionne de mépriser les déterminations individuelles pour mieux déchiffrer la maladie en son évolution typique - si bien que toute clinique orientée par un Kraepelin finira par menacer d'effacement les caractéristiques particulières et, partant, mépriser les indices subjectifs (Lepoutre, 2014); alors qu'à un pôle plutôt idiographique, Freud ne fera que chercher, même dans le symptôme le plus typique, la marque idiosyncrasique, par laquelle " sur un fond uniforme, chacun des malades appose ses conditions individuelles, ses lubies, pourrait-on dire, qui se contredisent directement dans chacun des cas " (Freud, 1916-17, p. 279) - en fait exactement comme le peintre de Morelli apposait au coin de son tableau sa marque inaliénable propre.

Cela donne comme résultats, dans l'écriture clinique, d'un côté un portrait typique, le psychiatre kraepelinien épurant une silhouette caractéristique et dessinant les contours d'une "personne abstraite " ${ }^{[6]}$ - quitte à composer schématiquement, et à des fins pédagogiques comme le fait le DSM, un portrait composite volontiers brossé à partir de plusieurs cas isolés, à la manière d'une photographie de Galton ; de l'autre, à l'inverse, une écriture où plus le texte du symptôme est singulier, plus il est significatif - ce pourquoi est mis en valeur, sur le mode du récit le plus détaillé,

[6] Voir à ce sujet l'excellente analyse d'Yvonne Wübben (2012) sur le paradigme «optique » de Kraepelin puisé dans la photographie composite, et d'une façon générale, la « rhétorique » kraepelinienne. 
le symptôme isolé comme ce que le sujet a de plus réel, sur le fond d'une histoire individuelle.

De là la conscience de Freud d'inventer une clinique inédite, en regard de la clinique psychiatrique classique. C'est ici, en effet, que l'on rencontre la nécessité du récit qui rend au malade la parole, le fait auteur de son histoire et sujet de son symptôme : c'est cette attribution qui modifie - radicalement - l'objet (le sujet) de la clinique freudienne, et lui propose son ancrage essentiel dans une clinique du sujet absolument particulier.

Faire ainsi cas du sujet implique de l'entendre dans son absolue singularité. Mais encore faut-il finalement entendre à quel niveau cette singularité prend son efficacité : non pas tant dans l'histoire, qui peut bien être indifférente dans l'originalité de son vécu et inefficace dans la linéarité de son déroulement manifeste, que dans la forme individuelle du symptôme où le sujet a réellement écrit sa marque, et qui recèle le véritable trait du cas.

Car il ne s'agit pas tant de retranscrire le récit du cas sous la dictée du patient, en s'en faisant le secrétaire passif, condamné à enregistrer et restituer comment le sujet (se) raconte son histoire pour y inscrire son symptôme - le vrai texte n'est pas là, tout fait, dans l'histoire racontée. Il tient plutôt dans la fidélité au fait clinique, laquelle ne peut être réalisée justement qu'en déjouant les pièges de l'imaginaire du sens, de la linéarité biographique, ce qui suppose de prendre acte que « toute mise en récit a une propension historiciste et masque, par le sens joui de la narrativité, la portée réelle de la structure et du cas clinique ”(Guerin, 2006, p. 65).

C'est pourquoi l'ambition qui oriente la clinique freudienne porte in fine derrière le récit qui fait sens : c'est bien plutôt, pour Freud, le symptôme lui-même qui doit $s$ 'écrire et prendre part à la conversation. Entendons : si le symptôme manifeste porte la trace de son processus de formation, s’il énonce lui-même les conditions de son apparition, c'est qu'il comporte en lui-même une narrativité intrinsèque. Bref, si le symptôme $a$ une histoire, il est lui-même une histoire qu'il faut savoir lire pour pouvoir la soutenir : c'est ce qui lui donne, à l'occasion, son aspect de scène - et impose du même coup à l'analyste, s’il veut s'en faire en quelque sorte " narratologue ", d'“ entrer dans tous les détails » (Freud, 1916-17, p. 269) du symptôme, pour en déplier l'étoffe historique.

\section{Références}

Assoun, P.-L. (1987). Freud, romancier du symptôme: sur l'écriture clinique freudienne. In Stroeken, H. (1987). En analyse avec Freud. Paris : Payot, pp. 7-41.

Engstrom, E. (2003). Bedside Science : Clinical Research in Heidelberg. In Clinical Psychiatry in Imperial Germany. London : Cornell University Press, pp. 121-146.

Freud, S. (1895). Études sur l'hystérie. In Freud, S. (2009). Eusres complètes, t. II. Paris : Puf.

Freud, S. (1905). Fragment d'une analyse d'hystérie. In Freud, S. (2006). Euvres complètes, t. VI. Paris : Puf. 
Freud, S. (1910). De la psychanalyse: cinq leçons données pour la célébration du vingtième anniversaire de la fondation de la Clark University de Worcester, Mass, septembre 1909. In Freud, S. (2009). Euvres complètes, t. X. Paris : Puf.

Freud, S. (1912). Conseils au médecin dans le traitement psychanalytique. In Freud, S. (2005). Euvres complètes, t. XI. Paris : Puf.

Freud, S. (1914). Le Moïse de Michel-Ange. In Freud, S. (2005). Euvres complètes, t. XII. Paris : Puf,. Freud, S. (1916-17). Leçons d'introduction à la psychanalyse. In Freud, S. (2000). Eusres complètes, t. XIV. Paris : Puf.

Freud, S. (2007). Lettres à Wilhelm Fließ. Paris : Puf.

Freud, S. \& Bernays, M. (2011). Die Brautbriefe. Frankfurt: S. Fischer, t. I.

Freud, S. \& Jung, C. G. (1975). Correspondance 1906-1914. Paris : Gallimard.

Ginzburg, C. (1980). Morelli, Freud and Sherlock Holmes. Clues and Scientific Method. In History Workshop, vol. 9, pp. 5-36.

Guérin, N. (2015). L'avarice du réaliste, le cas et la cause. In Faire cas de la clinique. Paris : Association de psychanalyse Encore, pp. 53-74.

Hirschmüller, A. (1991). Freuds Begegnung mit der Psychiatrie. Von der Hirnmythologie zur Neurosenlehre. Tübingen : Diskord.

Kohn, M. (1998). Le récit dans la psychanalyse. Paris : Érès.

Kraepelin, E. (1895). Über die Wachabteilung der Heidelberger Irrenklinik. In Allgemeine Zeitschrift für Psychiatrie, vol. 51, pp. 1-21.

Kraepelin, E. (1901). Die Heidelberger Wachabteilung für unruhige Kranke. In Zentralblatt für Nervenheilkunde (Neue Folge), vol. 12, pp. 705-713.

Kraepelin, E. (1902). Über die Wachabteilung der Heidelberger Irrenklinik. In Allgemeine Zeitschrift für Psychiatrie, vol. 59, pp. 133-136.

Lepoutre, T. (2014). Déconstruire Kraepelin : considérations historiques sur la nosologie kraepelinienne. In L'Évolution Psychiatrique, 79 (2), pp. 239-260.

Lepoutre, T., \& Villa, F. (2016). Le diagnostic à l'épreuve du traitement psychanalytique. In Cliniques Méditerranéennes, 94 (2), pp. 225-17.

Morelli, G. (1994). De la peinture italienne. Les fondements de la théorie de l'attribution en peinture à propos de la collection des galeries Borghese et Doria-Pamphili. Paris : Éditions de la Lagune.

Schor, N. (1980). Le détail chez Freud. In Littérature, vol. 37, nº 1, pp. 3-14.

Vanier, A. (2005). À propos de "psychiatrie et psychanalyse». In Psychologie clinique, n 20, pp. 77-84.

Villa, F. \& Fédida, P. (1999). Le cas en controverse. Paris : Puf.

Wind, E. (1989). Art et anarchie. Paris : Gallimard.

Wübben, Y. (2012). Verrückte Sprache. Psychiater und Dichter in der Anstalt des 19. Jahrhunderts. Konstanz: Konstanz University Press. 